\title{
Delay between pregnancy confirmation and sickle cell and thalassaemia screening: a population-based cohort study
}

\author{
Elizabeth Dormandy, Martin C Gulliford, Erin P Reid, Katrina Brown \\ and Theresa M Marteau on behalf of the SHIFT research team
}

\section{ABSTRACT}

\section{Background}

Antenatal sickle cell and thalassaemia screening sometimes occurs too late to allow couples a choice regarding termination of affected fetuses. The target gestational age for offering the test in the UK is 10 weeks.

Aim

To describe the proportion of women screened before 70 days' (10 weeks') gestation and the delay between pregnancy confirmation in primary care and antenatal sickle cell and thalassaemia screening.

\section{Design of study}

Cohort study of reported pregnancies.

\section{Setting}

Twenty-five general practices in two UK inner-city primary care trusts offering universal screening.

\section{Method}

Anonymised data on all pregnancies reported to participating general practices was collected for a minimum of 6 months.

Results

There were 1441 eligible women intending to proceed with their pregnancies, whose carrier status was not known. The median (interquartile range [IQR]) gestational age at pregnancy confirmation was 7.6 weeks (6.0-10.7 weeks) and $74 \%$ presented before 10 weeks. The median gestational age at screening was 15.3 weeks (IQR $=12.6-18.0$ weeks), with only $4.4 \%$ being screened before 10 weeks. The median delay between pregnancy confirmation and screening was 6.9 weeks

(4.7-9.3 weeks) After allowing for practice level variation, there was no association between delay times and maternal age, parity, and ethnic group.

\section{Conclusion}

About $74 \%$ of women consulted for pregnancy before 10 weeks' gestation but fewer than $5 \%$ of women were screened before the target time of 10 weeks. Reducing the considerable delay between pregnancy confirmation in primary care and antenatal sickle cell and thalassaemia screening requires methods of organising and delivering antenatal care that facilitate earlier screening to be developed and evaluated.

\section{Keywords}

Anaemia; genetic screening; pregnancy; primary health care; sickle cell; thalassaemia.

\section{INTRODUCTION}

Inherited disorders of haemoglobin are the most common disorders caused by single-gene defects, with $7 \%$ of the world's population being carriers and up to half a million births each year affected by severe forms of the disease. ${ }^{1}$ The 59th World Health Assembly recently urged member states to implement equitable and effective programmes for the prevention and management of sickle cell anaemia. ${ }^{2}$ In the UK, following the NHS Plan in 2000, an antenatal Sickle Cell and Thalassaemia Programme is currently being implemented with the aim of offering timely antenatal sickle cell and thalassaemia screening to all women to facilitate informed decision making. ${ }^{3}$ This is supported by guidelines stating that the screening process should be completed before the end of the first trimester, ${ }^{4,5}$ which means that screening should take place before 10 weeks' gestation and that prenatal diagnosis and any subsequent action should take place before the end of the 12 weeks' gestation. ${ }^{3}$

However, some evidence suggests that the screening process does not facilitate timely

E Dormandy, PhD, research fellow; EP Reid, $M S c$, research assistant; K Brown, $M S c$, research assistant; TM Marteau, PhD, Cpsychol FMedSci, professor of health psychology, Department of Psychology (at Guy's), Health Psychology Section, Institute of Psychiatry; MC Gulliford, FRCP, FFPH, professor of public health, Department of Public Health Sciences, King's College London, London.

Address for correspondence

Professor Theresa M Marteau, 5th Floor, Thomas Guy House, Guy's Campus, King's College, London Bridge, London SE1 9RT. E-mail: theresa.marteau@kcl.ac.uk

Submitted: 5 June 2007; Editor's response: 14 September 2007; final acceptance: 26 September 2007.

(C) British Journal of General Practice 2008; 58: 154-159. DOI: $10.3399 /$ bjgp08X277267 
informed decisions because tests are sometimes offered too late for some women to allow them the full range of reproductive options, either following their reluctance to undergo invasive prenatal testing late in pregnancy $^{6-8}$ or because abortion laws may preclude termination of affected pregnancies later in pregnancy.

A recent audit in the UK found that only 11 out of 59 couples at risk of a significant sickle cell or thalassaemia disorder were offered antenatal screening before the end of the first trimester (Newham Sickle Cell and Thalassaemia Centre, unpublished data, 2007).

Any delay between pregnancy confirmation and the opportunity for screening has the potential to undermine the policy and practice of offering antenatal sickle cell and thalassaemia screening early in pregnancy to facilitate timely reproductive choice for women. The extent of this delay is unknown in population-based data. Timely reproductive choice can also be undermined by the timeliness of reporting results, counselling, and prenatal diagnosis. Data on the likely extent of these delays are available ( $\mathrm{J}$ Behrens et al, unpublished data, 2003)..$^{6,9-11}$

The study presented here estimates the extent to which women who are pregnant have access to timely screening. The delay between pregnancy confirmation in primary care and time of antenatal sickle cell and thalassaemia screening is reported here using population-based data, and examines the extent to which patient and practice characteristics are associated with longer delays.

\section{METHOD}

A cohort study was conducted of all pregnancies reported in 25 general practices from two primary care trusts (PCTs) in the UK. The two PCTs are ranked among the most deprived in England (sixth and 13th out of 354 boroughs) and have about $40 \%$ of their total populations from minority ethnic groups. ${ }^{12}$ The study represented the run-in phase of a cluster randomised controlled trial of three methods of offering antenatal sickle cell and thalassaemia screening in primary care, that is, before randomisation of the practices in the trial. ${ }^{13}$

All general practices in two PCTs (123 general practices) were sent a written invitation to take part in a trial examining the feasibility, effectiveness and acceptability of offering antenatal sickle cell and thalassaemia screening in primary care. Thirty-two practices expressed an interest in taking part and 27 agreed to participate in the trial. Two practices subsequently withdrew. The list sizes of participating practices ranged from 1300 to 15000 . The participating practices did not differ from the

\section{How this fits in}

Women's choices about prenatal diagnostic testing for sickle cell or

thalassaemia are restricted because diagnostic tests are offered too late in

pregnancy to allow reproductive choice. It is unclear whether such tests are

offered late because women delay confirming their pregnancies in primary care

or because there is a health service delay in offering antenatal sickle cell and

thalassaemia screening. This study shows that women do not delay confirming

their pregnancies. A delay of 7 weeks was observed between women

confirming their pregnancies in primary care and undergoing sickle cell and

thalassaemia screening.

other practices in the two PCTs with respect to the proportion of single-handed GPs $(P=0.33)$, the list size per GP $(P=0.99)$, the Townsend score $(P=$ $0.69)$ or the resident percentage of minority ethnic groups $(P=0.80)$.

Data were collected during 2005-2006 at each practice for a minimum of 6 months with the intention of providing data on at least 33 pregnancies. If necessary, the data collection period was extended until data were available on 33 pregnancies. A universal screening policy was operating during the data-collection period; that is, antenatal sickle cell and thalassaemia screening was offered to all women who were pregnant, regardless of the couple's ethnic group or family origin. ${ }^{3}$ Data collected from the 25 participating practices is reported here.

Anonymised data were collected for the date of the last menstrual period, the date of the pregnancy confirmation visit in primary care, and the date of the test, as defined by the date of venesection. These were used to calculate:

- gestational age at time of pregnancy confirmation in primary care; and

- gestational age at time of sickle cell and thalassaemia screening.

Screening uptake was ascertained up to 26 weeks' gestation. Women who were not tested before 26 weeks' gestation were classified as not tested for the purpose of prenatal screening of the foetus. Routinely collected data for date of birth, parity, ethnic group, previously determined carrier status, termination of pregnancy, or miscarriage were also extracted from primary care computer systems. Completeness of the number of pregnancies reported at each practice was checked by comparing the number of pregnancies reported with the number of maternity referrals made by each practice. The date of antenatal sickle cell and thalassaemia screening was 
obtained from maternity units and laboratories at hospitals to which the women were referred for maternity care.

Data for times were skewed and are reported as medians (interquartile ranges [IQRs]). The distribution for the median delay for each participating general practice was evaluated. A multiple linear regression model was implemented with the delay between pregnancy confirmation visit and screening for each patient as the dependent variable. Explanatory variables were maternal age (continuous), parity (primiparous, multiparous, and not known), and ethnic group (north European, south and other European, Black African and African Caribbean, south and southeast Asian, other, mixed, and not known). For analysis, women classified as south and other European, Black African and African Caribbean, south and south-east Asian, other, and those from mixed ethnic groups were banded together as 'higher risk'.

A random effects model with maximum likelihood estimation, fitted using the 'xtreg' command in Stata (version 9), was used to allow for clustering by practice.

\section{RESULTS}

There were 2062 pregnancies across the 25 practices that completed the run-in data collection phase. Women were omitted from the analysis if the date of the last menstrual period was not known $(n=62)$, if the carrier status was already known $(n=299)$, if the woman had a termination of pregnancy for reasons other than fetal abnormality ( $n=157)$, if there was a miscarriage $(n=117)$, or if pregnancies were confirmed before 26 weeks gestation.

Figure 1. Distributions for gestational age at first pregnancy confirmation and sickle cell and thalassaemia screening for women whose

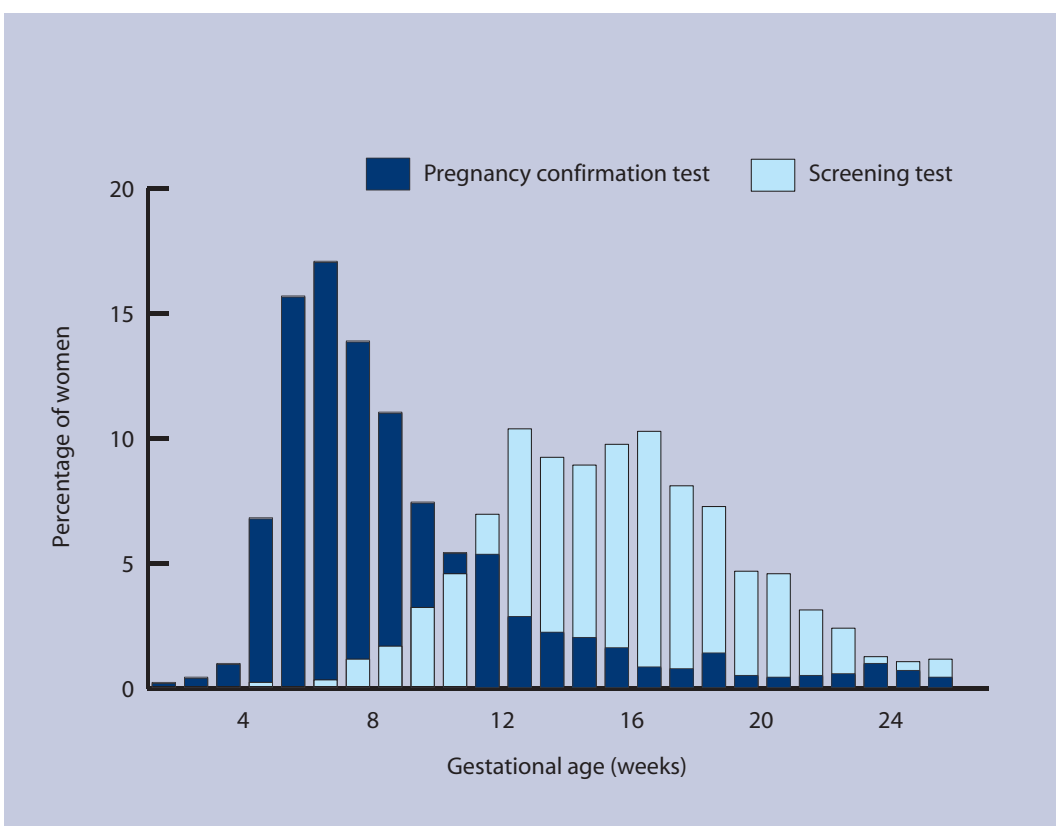

the pregnancy confirmation visit was after 26 weeks' gestation $(n=126)$. Some women were excluded on more than one criterion. There were 1441 eligible women, whose carrier status was not known, who intended to proceed with the pregnancy and whose pregnancy confirmation visit was before 26 weeks' gestation.

The women's median age at the last menstrual period was 28.5 years (IQR $=24.0-33.5$ years) and 767 women (53\%) were primiparous. Routinely recorded data for ethnic group, obtained from practices, were available for 837 (58\%) women. In total, 645 (45\%) women were from 'higher-risk' groups: Black African and African Caribbean ( $n=$ $228)$, south and other European $(n=99)$, south and south-east Asian ( $n=260)$, other $(n=47)$, and mixed $(n=11)$. The median gestational age at the pregnancy confirmation visit in primary care for all women was 7.6 weeks (IQR $=6.0-10.1$ weeks). There were 1060 (74\%) women whose pregnancy confirmation was before 70 days' (10 weeks') gestation. The gestational age at the pregnancy confirmation visit for the 965 (67\%) women who were screened before 26 weeks was 7.4 weeks (IQR = 5.9-9.9 weeks). Among women whose reported ethnic group data were available, there was a weak association between ethnic group and gestational age at booking after adjusting for maternal age, parity, and clustering $(P=0.023)$. The median gestational age at booking ranged from 7.1 weeks in north European women to 8.4 weeks in south and other Europeans.

From the 1441 pregnancies eligible for analysis there were 965 (67\%) women who had screening tests performed before 182 days' (26 weeks') gestation. The proportion screened did not vary by ethnic group. The median gestational age at screening was 15.3 weeks (IQR $=12.6-18.0$ weeks), with 63 (4.4\%) being screened before 10 weeks' gestation (Figure 1). The median delay between pregnancy confirmation in primary care and antenatal sickle cell and thalassaemia screening in these women was 6.9 weeks (IQR $=4.7-9.3$ weeks).

Variation between practices was greater than expected by chance $(P<0.001)$. At the practice with the shortest delays, the median time from pregnancy confirmation in primary care to screening was 3.7 weeks; at the practice with the highest delay, the median time from pregnancy confirmation in primary care to screening was 10.0 weeks. After allowing for practice level variation, there was no association between delay times and the measured individual patient characteristics including maternal age, parity, and ethnic group. Specifically, women's ethnic group was not associated with delay times and variation 
between practices was not accounted for by adjusting for ethnic group. In addition, there was no association between ethnic group category and gestational age at screening $(P=0.351)$ or with the proportion screened by 26 weeks $(P=0.060)$.

\section{DISCUSSION}

\section{Summary of main findings}

These population-based data show a 7-week delay between pregnancy confirmation in primary care and antenatal sickle cell and thalassaemia screening. This undermines the policy and practice of facilitating informed reproductive choices early in pregnancy. These data are supported by a small retrospective study of women at risk of a sickle cell disorder that showed that some women did not have screening until 15 weeks' gestation, many weeks after reporting their pregnancies in primary care. ${ }^{7}$ Furthermore, a third of women across all ethnic groups reported their pregnancies to their GPs early in pregnancy but did not undergo sickle cell and thalassaemia screening before 26 weeks' gestation. The data collected do not indicate whether this represents a service failure or individual choice not to undergo screening. Further work is needed to understand why a third of women were not tested.

Even in this deprived inner-city setting, women generally attended their general practices early in pregnancy for confirmation. The delay before screening uptake was not associated with individual women's age, parity, or ethnic group. There was, however, considerable variation in the extent of delays between general practices with the best-performing practices having a median delay of just over 3 weeks from pregnancy confirmation in primary care to screening. Practice level variation may result from primary care delays in referring women for antenatal care or secondary care delays in acting on these referrals. Individual patient factors did not appear to be associated with delays in screening uptake.

Targets specifying that the screening process, including any offer of prenatal diagnostic testing, should be completed before the end of the first trimester are clearly not yet being met, with only $4.4 \%$ of women undergoing screening before their 10 th week of pregnancy. The results suggest that there is considerable scope for facilitating timely screening through improved organisation and delivery of antenatal care.

\section{Comparison with existing literature}

It has been suggested that the delay between pregnancy confirmation in primary care and the first midwifery appointment is greater for women from minority ethnic groups than for other women, ${ }^{14-16}$ Although there was some evidence that women from some minority ethnic groups attend primary care to confirm their pregnancies approximately 1 week later than other women, there was no evidence that the 7-week delay between pregnancy confirmation and testing was associated with ethnic group. This indicates that delays within the health service, rather than women reporting late to confirm their pregnancies, are responsible for the large observed delay in testing.

\section{Strengths and limitations of the study}

This is the first study to examine the delay between pregnancy confirmation in primary care and antenatal sickle cell and thalassaemia screening using population-based data. There are, however, some limitations concerning the generalisability of the results.

The general practices observed were not randomly selected from the 123 general practices in the two PCTs studied and, therefore, may not be representative of the practices in these PCTs. The variables on which data are available show that participating practices were similar to other practices in the study PCTs in relation to practice size, deprivation, and patient ethnic group. In addition, the study PCTs were chosen to represent geographical areas with a high prevalence of sickle cell and thalassaemia disorders and, therefore, may not be representative of other areas. They represent deprived inner-city areas where general practice lists are longer than those in suburban or rural areas and primary care services are generally less well organised. ${ }^{17}$

Ethnic group data were not collected systematically across the practices and were available for about half of the women. This reflects a shortfall in the collecting of basic demographic data in primary care.

Finally, and related to the last point, the accuracy of the collected data was dependent on the reliability and validity of the data held in GP record systems. Following fidelity checks on the number of pregnancies, the data presented here are an accurate representation of the number of pregnancies. The reliability of data collected about these pregnancies is not known.

\section{Implications for clinical practice}

The results suggest that there is considerable scope for facilitating timely screening through improved organisation and delivery of antenatal care. One option currently being evaluated in the SHIFT (Screening for Haemoglobinopathies In First Trimester) trial is for GPs to offer tests as pregnancy 
is confirmed in primary care..$^{13}$ Offering antenatal screening in primary care would, however, require a considerable change in practice for GPs and midwives. It may be that such a change could be best achieved by midwives and GPs working more closely together; an alternative is to consider rapid referral systems to midwives for women who are pregnant.

The considerable delay between pregnancy confirmation in primary care and antenatal sickle cell

\section{COMMENTARY}

\section{Important role for primary care in ensuring informed choice and timely screening and care}

Sickle cell disease is now as common as cystic fibrosis in England and is one of the commonest reasons for admission to hospital in London. ${ }^{1,2}$ The paper by Dormandy and colleagues reports that a cohort of women in a deprived inner-city setting confirmed their pregnancy early on (median gestation 7.6 weeks). ${ }^{3}$ Despite this early confirmation, antenatal sickle cell and thalassaemia testing did not occur for many weeks (median gestation 15.3 weeks). These results raise two problems. First, services in their present form are falling a long way short of the NICE guidelines that to achieve truly informed choice women should be offered antenatal sickle cell and thalassaemia screening by 10 weeks gestation; and second, the failure to test early in pregnancy results mostly from an NHS failure to deliver early testing rather than from women failing to report their pregnancy early.

So what are the possible ways forward to ensure that all pregnant women have access to good quality antenatal sickle cell and thalassaemia screening in a timely manner? One solution would be to offer antenatal sickle cell and thalassaemia screening in primary care when women first report their pregnancy. Given the findings reported here, this method would be likely to achieve earlier testing for many women. Such a change, as well as requiring changes in general practice and midwifery care, would be difficult because antenatal sickle cell and thalassaemia screening could be perceived as somehow different from other aspects of maternity care.

An alternative way forward would be to acknowledge that sickle cell and thalassaemia screening could be considered as a test for life rather than an antenatal test. It could be conducted in primary care at any stage, not just when the woman is pregnant. To ensure joined-up care the carrier test result could be included on the maternity referral form, much as relevant history is now included.

In the wider view this would fit well with the development of a coherent preconception screening and care policy offered to women and couples as part of young adult checks, contraceptive care, and pre-pregnancy planning. ${ }^{4}$ The findings of Locock and Kai show that parents would welcome an sickle cell and thalassaemia screening programme before pregnancy. ${ }^{5}$ At present such a policy and service is lacking from the NHS despite the obvious benefits it would provide. In the case of sickle cell and thalassaemia screening countries such as Cyprus and Iran who offer preconception programmes, and in the case of Iran have even passed a fatwa to allow prenatal diagnosis and termination early in pregnancy, have achieved most success. ${ }^{6} \mathrm{~A}$ national preconception screening and care programme would help to clarify that the current 'turf war' between midwifery and primary care is unhelpful and would demonstrate the important role for primary care as key to the long-term care of women and their families, which starts before pregnancy and continues through childhood and often into the next generation.

The data reported here highlight an urgent need for action, and the debate about how to achieve change should not lose sight of the need to achieve change. An important area for general practice to address is the lack of knowledge in primary care about the need for early antenatal testing, which patients will increasingly expect to be routinely provided in this setting. ${ }^{6}$ The experience of the PEGASUS (Professional Education for Genetic Assessment and Screening) programme commissioned by the NHS Sickle Cell and Thalassaemia Screening Programme is that, to date, primary care has been a difficult audience to engage.

\section{Allison Streetly}

Programme Director, NHS Sickle Cell \& Thalassaemia Screening Programme, King's College London, Division of Health and Social Care, Department of Public Health Sciences, 6th Floor, Capital House, 42 Weston Street, London SE1 3QD.

\section{REFERENCES}

1. London Health Observatory. Analysis of frequent hospital users, by PCT 2003/4. http://www.lho.org.uk/viewResource.aspx?id=10286 (accessed 17 Jan 2008 ).

2. Streetly A, Clarke M, Downing M, et al. Implementation of the universal newborn screening programme for sickle cell disease in England: results for $2003-2005$. $J$ Med Screening (in press).

3. Dormandy E, Gulliford M, Reid EP, et al. Delay between pregnancy confirmation and sickle cell thalassaemia screening: a population-based cohort study. Br J Gen Pract 2008; 58: 154-159.

4. Chapple J. Preconception care (National Knowledge week). http://www.library.nhs.uk/screening/ViewResource.aspx?resID=269308 (accessed 17 Jan 2008 ).

5. Locock L, Kai J. Parents' experiences of universal screening for haemoglobin disorders: implications for practice in new genetics era. Br J Gen Pract 2008; 58: 161-168.

6. Samavat A, Modell B. Iranian national thalassaemia screening programme. BMJ 2004; 329: 1134-1137.

7. Qureshi N, Armstrong S, Modell B. GPs' opinions of their role in prenatal genetic services: a cross-sectional survey. Fam Pract 2006, 23(1): 106-110. 
and thalassaemia screening may deprive couples of opportunities to opt for prenatal diagnosis early in pregnancy. The results of the current study suggest that the cause and solution to this delay lies in healthcare delivery.

\section{Funding body}

This study is funded by the UK Department of Health through its Health Technology Assessment Programme, (reference number 03/02/03). Antenatal screening for haemoglobinopathies in primary care: a cluster randomised trial to inform a simulation model (Acronym: SHIFT [Screening for Haemoglobinopathies In First Trimester])

\section{Ethics committee}

Ethical approval was granted to collect anonymised data on all pregnancies reported in participating practices (05/Q0501/36)

\section{Competing interests}

The authors have stated that there are none. The opinions and conclusions expressed here are those of the authors and do not necessarily reflect those of the UK NHS or the Department of Health

\section{Acknowledgements}

We are very grateful to the general practices who participated in this study. This paper was written on behalf of the SHIFT research team (AE Ades, Mike Calnan, MRC Health Service Research Collaboration; Elizabeth N Anionwu, Thames Valley University; Karl Atkin, University of York; Stirling Bryan, Tracy Roberts, University of Birmingham; Verna Davis, Manchester Sickle Cell \& Thalassaemia Centre; Moira Dick, Lambeth PCT, London; Hilary Harris, Brooklands Medical Practice; Tracy Johnston, St Mary's Hospital for Women \& Children, Manchester; Patricia Jones, Barbara Wild, University College Hospital, London; Jon Karnon, University of Sheffield; Fred Kavalier, Guy's Hospital, London; Jane Logan, Mawbey Brough Health Centre, London).

\section{Discuss this article}

Contribute and read comments about this article on the Discussion Forum: http://www.rcgp.org.uk/bjgp-discuss

\section{REFERENCES}

1. Weatherall D, Akinyanju O, Fucharoen S, et al. Inherited disorders of hemoglobin. In: Jamieson DT, Mosely WH, Measschon AR (eds). Disease control priorities in developing countries. (2nd edn). Oxford: Oxford University Press and The World Bank, 1993: 663-680.

2. World Health Organisation. 59th World Health Assembly Agenda, Item 11.4. Geneva, Switzerland: WHO, 2006 www.who.int/gb/ebwha/pdf_files/WHA59/A59_R20-en.pdf (accessed 25 Jan 2008).

3. NHS Sickle Cell and Thalassaemia Screening Programme. Standards for linked antenatal and newborn screening programme. London: UK National Screening Programme, King's College, 2006. http://www.sct.screening.nhs.uk/Documents/ProgrammeSTAN.pdf (accessed 25 Jan 2008).

4. National Institute for Clinical Excellence. Antenatal care: routine care for the healthy pregnant woman. Clinical Guideline 6. London: RCOG press, 2003.

5. Department of Health. National Service Framework for Children, young people and maternity services: maternity services. London: Department of Health, 2004.

www.dh.gov.uk/en/Publicationsandstatistics/Publications/PublicationsP olicyAndGuidance/DH_4089101 (accessed 25 Jan 2008)

6. Modell B, Harris R, Lane B, et al. Informed choice in genetic screening for thalassaemia during pregnancy: audit from a national confidential inquiry. BMJ 2000; 320(7231): 337-341.

7. Neuenschwander H, Modell B. Audit of process of antenatal screening for sickle cell disorders at a north London hospital. BMJ 1997; 315(7111): 784-785.

8. Thomas P, Oni L, Alli M, et al. Antenatal screening for haemoglobinopathies in primary care: a whole system participatory action research project. Br J Gen Pract 2005; 55(515): 424-428.

9. Wright J, Rati N, Kennefick A, et al. A pilot study of 'fast track' antenatal screening for haemoglobinopathies. J Med Screen 2003; 10(4): 169-171.

10. Gill C, Klynman N. Care Pathways for antenatal and neonatal haemoglobinopathy screening in Greater London, 2003. NHS Sickle Cell and Thalassamia Programme.

http://www.sct.screening.nhs.uk/Documents/LondonMappingReport.p df (accessed 25 Jan 2008)

11. Greengross P, Hickman M, Gill M, et al. Outcomes of universal antenatal screening for haemoglobinopathies. J Med Screen 1999; 6(1): $3-10$.

12. Office for National Statistics. Neighbourhood statistics. Indices of deprivation 2004: Local authority summaries. London: National Statistics.

http://www.neighbourhood.statistics.gov.uk/dissemination/LeadTableVi ew.do? $\mathrm{a}=7 \& \mathrm{~b}=276767 \& \mathrm{c}=$ newham $\& \mathrm{~d}=13 \& \mathrm{e}=10 \& \mathrm{~g}=343478 \& \mathrm{i}=1001 \mathrm{x}$ $1003 \times 1004 \& \mathrm{o}=35 \& \mathrm{~m}=0 \& \mathrm{r}=1 \& \mathrm{~s}=1201083025343 \& \mathrm{enc}=1 \& \mathrm{dsFamilyId}$ $=724$ (accessed 25 Jan 2008).

13. Marteau TM, Ades T, Anionwu EA, et al. Antenatal screening for haemoglobinopathies in primary care: a cluster randomised trial to inform a simulation model (SHIFT), 2004. http://www.hta.ac.uk/project/1401.asp (accessed 25 Jan 2008).

14. Rowe RE, Garcia J. Social class, ethnicity and attendance for antenatal care in the United Kingdom: a systematic review. J Public Health Med 2003; 25(2): 113-119.

15. Ahmad W, Atkin K. Primary care and haemoglobin disorders: a study of family and professionals. Critical Public Health 2000; 10(1): 41-53.

16. Green J, Dawson MF. Women's experience of screening in pregnancy: ethnic differences in the West Midlands. In: Clarke A, Parsons E (eds). Culture, kinship and genes: towards cross-cultural genetics. Basingstoke: MacMillan, 1997: 120-133

17. Adams G, Gulliford M, Ukoumunne O, et al. Geographical and organisational variation in the structure of primary care services: implications for study design. J Health Serv Res Policy 2003; 8(2): 87-93. 